TP Periodica Polytechnica Electrical Engineering and Computer Science

59(3), pp. 88-93, 2015

DOI: $10.3311 /$ PPee. 8576

Creative Commons Attribution (i)

RESEARCH ARTICLE

\section{Resonance Analysis of a Transmission Power System and Possible Consequences of its Undergrounding}

\author{
Olga Galland $^{1 *}$, David Leu ${ }^{1}$, Valentin Berner ${ }^{1}$, Patrick Favre-Perrod ${ }^{1}$
}

Received 13 May 2015; accepted after revision 21 August 2015

\begin{abstract}
Presently there is a great interest in underground cable transmission lines in extra high and high voltage level networks. The undergrounding of the transmission system can lead to the decrease of the resonance frequencies resulting in high overvoltages and possible power system component damages. It is therefore necessary to analyze possible resonance problems in the mixed power transmission system. In this work a frequency dependent model for transmission systems is implemented and the frequency scan results are presented. In order to gain a better understanding of the nature and extent of the resonance, a resonance mode analysis is also executed. The proposed procedure is tested on two examples: a ten-node power system and the Swiss extra high voltage (EHV) power transmission system. Power systems comprising solely overhead lines and different variations of mixed power system are examined.
\end{abstract}

\section{Keywords}

Resonance frequencies, Power transmission, Resonance mode analysis, High-voltage cables, Power system harmonics
${ }^{1}$ School of Engineering and Architecture of Fribourg,

University of Applied Sciences and Arts of Western Switzerland,

CH-1705 Fribourg, P.O.B. 32, Switzerland

"Corresponding author, e-mail: Olga.Galland@hefr.ch

\section{Introduction}

Power transmission systems need to be expanded due to continuously rising demand and existing plans for increasing the generation share of renewable energy sources. Nowadays it is necessary to consider the importance of citizens' and environmental groups' opinions in the choice of the transmission line construction. There is therefore a high interest in transmission system undergrounding. At the same time the power system becomes more and more complex due to the increasing usage of power electronic components. Furthermore, overlay DC networks are increasingly considered as a network extension option. Electronic components are often sources of harmonics and normally act like frequency-dependent current sources exciting system resonance frequency. Throughout this paper resonance frequency is understood as parallel resonance frequency (impedance is at its maximum). Because of their capacitive behavior underground (UG) cables can lead to a drop in resonance frequency. These facts increase significantly the risk of overvoltage in the system, which may in turn cause severe damage to network components. To prevent these problems a frequency analysis of the power transmission system should be done.

There have been reports of resonance problems in various large scale power transmission systems. For example, resonances were detected and studied in the transmission system of SBB (Swiss Federal Railways). The studies were done for the frequency range up to $\sim 300 \mathrm{~Hz}$ (the fundamental frequency is $16.67 \mathrm{~Hz}$ ). Legacy clear track signaling systems still operate at $\sim 103 \mathrm{~Hz}$ (6th harmonic order) and no resonance is therefore allowed in the network at frequencies near $100 \mathrm{~Hz}$. However due to the increased utilization of UG cables (planed up to 355 $\mathrm{km}$ by 2025 ) this limit may be reduced to $\sim 87 \mathrm{~Hz}$ [1].

The risks related to power transmission undergrounding are discussed in [2-4]. In [5-7] the resonance mode analysis (RMA) method is presented. The authors discuss the characteristics of the eigenvalues in terms of the detection of the nature and extent of the resonance. The possible implementation of RMA is also pointed out. In [8] the authors compare two methods of analysis for network resonances: frequency scan and RMA. These methods are applied to a test system and then the 
RMA is implemented for one part of the German power transmission system. Scenarios of high (inductive behavior) and low load (capacitive behavior) conditions are presented. It is also concluded that the switching operations of any series or shunt power system component may dramatically influence the system resonance. Harmonic issues associated with a long underground cable in the transmission system are discussed in [9]. In [10] subsea power cables are considered. This paper shows the importance of the interactions between all of the power system components in harmonic studies of ac-dc systems. Frequency domain transformer models are elaborated in [11] and [12]. In [13] a review of the modeling of network components and analysis of harmonic propagations in power systems is illustrated.

The objective of this project is to analyze possible resonance problems in mixed power systems related to the addition of UG cables and to investigate the impact of different voltage levels on each other.

This paper is organized in five sections. Following the introduction, the methods and models implemented in this work are explained in Section 2. A case study is then introduced and the results obtained are discussed in Sections 3 and 4, respectively. Finally, conclusions are drawn in Section 5.

\section{Methods and models}

\subsection{Frequency scan method}

The frequency scan method is based on the nodal impedance matrix:

$$
\left[\begin{array}{c}
V_{1} \\
V_{2} \\
\vdots \\
V_{n}
\end{array}\right]=\left[\begin{array}{cccc}
Z_{11} & Z_{12} & \cdots & Z_{1 n} \\
Z_{21} & Z_{22} & \cdots & Z_{2 n} \\
\vdots & \vdots & \ddots & \vdots \\
Z_{n 1} & Z_{n 2} & \cdots & Z_{n n}
\end{array}\right]\left[\begin{array}{c}
I_{1} \\
I_{2} \\
\vdots \\
I_{n}
\end{array}\right] .
$$

The nodal impedance at any node is equivalent to the corresponding diagonal element of the nodal impedance matrix. It is therefore possible to obtain the frequency characteristics of the nodal impedance by performing a frequency scan of the related diagonal element of the nodal impedance matrix [14].

\subsection{Resonance mode analysis}

The resonance mode analysis (RMA) is another tool to obtain the frequency characteristics of the power system. Unlike frequency scan method RMA is based on the eigenanalysis and it therefore allows to determine impedances in the modal coordinate system. The nodal impedance matrix can be transformed into the modal impedance matrix:

$$
\left[Z_{m}\right]=[L]\left[\begin{array}{cccc}
Z_{11} & Z_{12} & \cdots & Z_{1 n} \\
Z_{21} & Z_{22} & \cdots & Z_{2 n} \\
\vdots & \vdots & \ddots & \vdots \\
Z_{n 1} & Z_{n 2} & \cdots & Z_{n n}
\end{array}\right][R],
$$

Where

$$
\left[Z_{m}\right]=\left[\begin{array}{cccc}
Z_{m_{1}} & 0 & \cdots & 0 \\
0 & Z_{m_{2}} & \cdots & 0 \\
\vdots & \vdots & \ddots & \vdots \\
0 & 0 & \cdots & Z_{m_{n}}
\end{array}\right],
$$

Sub-indices $n$ and $m$ are node and mode number, respectively. $[L]$ and $[R]$ are the left and the right eigenvector matrices, respectively [5]. Since phase shifting transformers are ignored the system admittance matrix is a symmetric matrix

$$
[L]=[R]^{-1}
$$

The modal impedance matrix is the inverse of the diagonal eigenvalue matrix:

$$
\left[Z_{m}\right]=[\Lambda]^{-1}=\left[\begin{array}{cccc}
\lambda_{1}^{-1} & 0 & \cdots & 0 \\
0 & \lambda_{2}^{-1} & \cdots & 0 \\
\vdots & \vdots & \ddots & \vdots \\
0 & 0 & \cdots & \lambda_{n}^{-1}
\end{array}\right]
$$

All the variables in the above stated equation are frequency dependent [5].

The participation factors of the nodes to the critical mode can be found [5]:

$$
P F_{n, m}=L_{m}^{n} R_{m}^{n}
$$

\subsection{Proposed model}

The model is prepared for a general power system. The overall procedure is carried out as shown on Fig. 1. The frequency range and nominal frequency need to be provided as input data by the user, and the algorithm determines the studied frequency interval at each step. In order to decrease the number of frequency steps used for frequency analysis, the frequency interval should be adjusted automatically i.e. it should depend on the difference between the last determined values of the impedance magnitude and phase and the reference ones. The values of the impedance magnitude and phase at the nominal frequency are used as the reference values.

To compute the admittance matrices for each voltage level (VL) it is necessary to determine the transmission line parameters at harmonic frequencies. In this paper two types of transmission lines are considered: overhead $(\mathrm{OH})$ lines and $U G$ cables. The following assumptions were made for the modelling of the transmission lines: the same type and configuration of pylon is used for all $\mathrm{OH}$ lines of the same voltage level; characteristics of transmission line corridors are considered to be the same all over the power system; the UG cables are buried at the same depth on their entire length. The following effects are included: frequency dependence of the line parameters, skin 
effect, long-line effect, line imbalances, and line transpositions. The procedure of the transmission line parameters determination is explained in [15] for overhead lines ( $\pi$-model) and in [14] and [16] for UG cables.

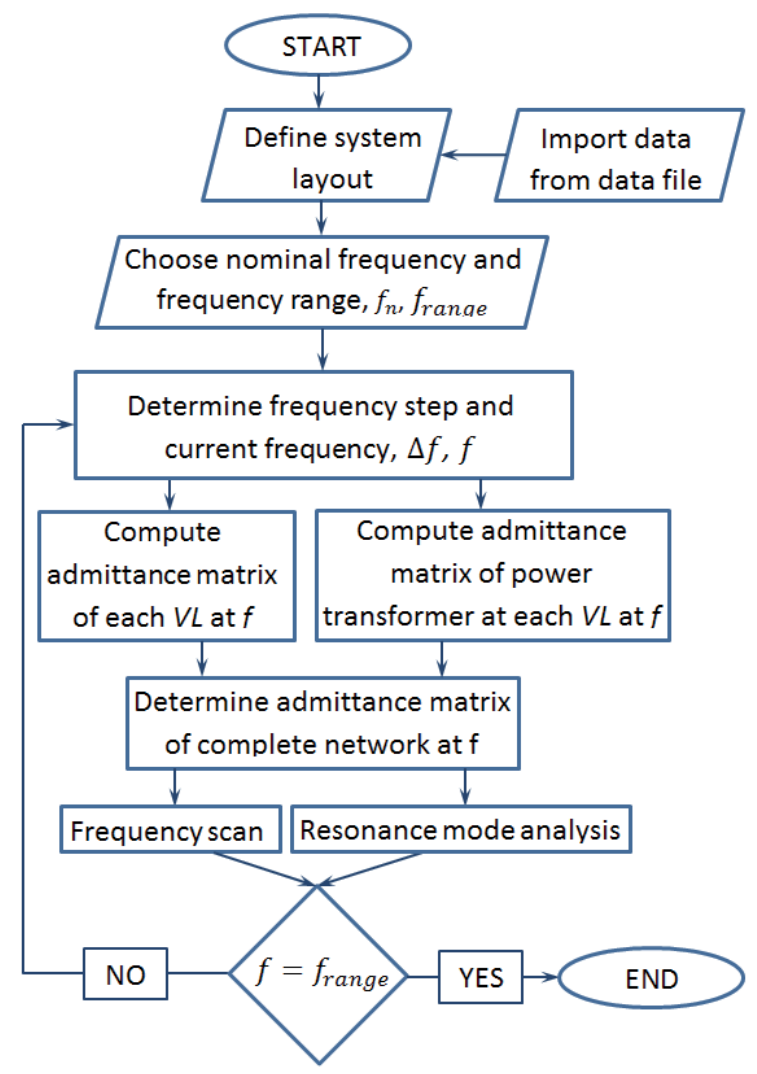

Fig. 1 Flowchart diagram of the proposed model

The power transformer is modeled with the following simplifications: since in this work the studied frequency range is up to $2 \mathrm{kHz}$ the capacitive effect and the saturation effect are neglected [17]. The skin effect is taken into account since it becomes important at harmonic frequencies. The modelling of the three-phase transformer is described in [15], [14], and [17].

\section{Case study}

Two power systems are used in this study: an imaginary ten-node system and the Swiss power transmission system $(380 / 220 \mathrm{kV})$.

\subsection{Ten-node system}

The ten-node system contains two voltage levels: $380 \mathrm{kV}$ and $60 \mathrm{kV}$, and a star-ground - star-ground transformer, Fig. 2. Each of these networks contains five nodes and five transmission lines.

The purpose of implementing a ten-node system is to show the difference in resonance frequency of the power system containing solely overhead lines and of the meshed power system comprising both overhead lines and underground cables. This example is also aimed at studying the possible interactions between two network levels. Four simulations were therefore done:
- case $\mathrm{A}$ - all transmission lines are $\mathrm{OH}$ lines;

- case B - line 3-5 (380 kV) changed to UG cable;

- case C - lines 2-3 and 3-5 (60 kV) changed to UG cable;

- case D - lines 3-5 (380 kV), 2-3 and 3-5 (60 kV) changed to UG cables.

The length of transmission lines remained the same in all studied cases.

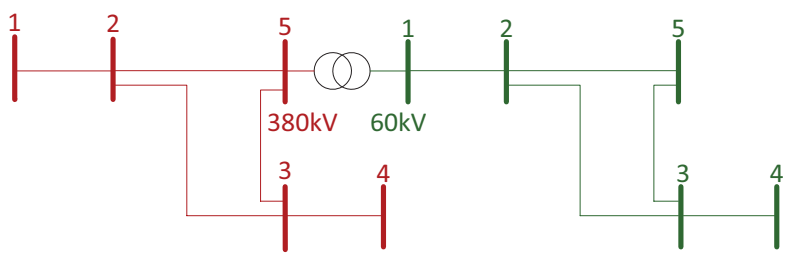

Fig. 2 Ten-node system

\subsection{Swiss EHV power transmission system}

The configuration of the Swiss EHV power transmission system is shown in Fig. 3.

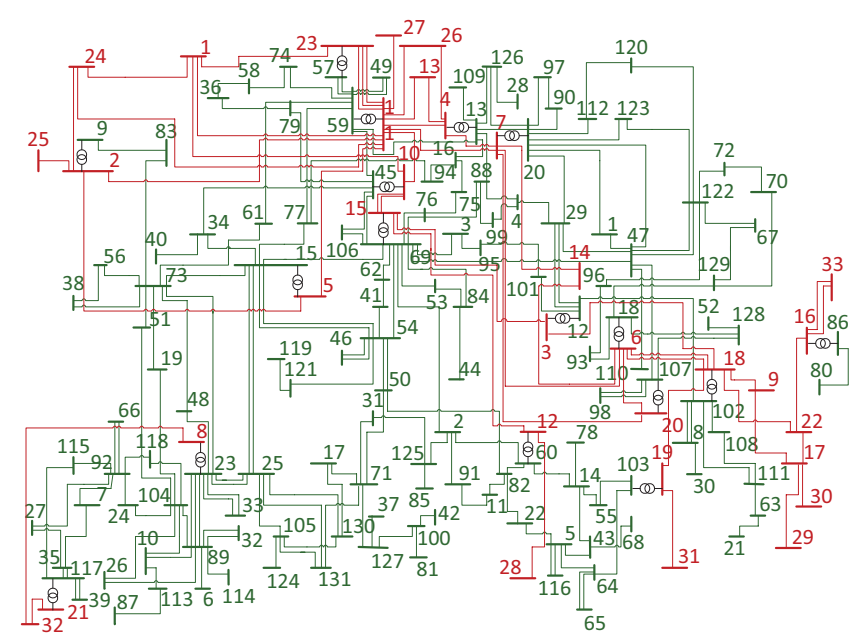

Fig. 3 Swiss EHV Power Transmission System: Red -380 kV, Green - 220 kV

This system comprises two voltage levels ( $380 \mathrm{kV}$ and 220 $\mathrm{kV})$. It consists of 164 nodes, 244 transmission lines, and 18 power transformers (only 380/220 kV transformers).

This example allows investigating the possible challenges related with undergrounding of transmission power system. The results of the following simulations are therefore presented in this paper:

- case $\mathrm{E}$ - the actual power system;

- case F - line 81-100 (220 kV) represented as UG cable (connection nodes have high PF);

- case $\mathrm{G}$ - line 31-71 (220 kV) represented as UG cable (connection nodes have medium PF);

- case H - line 23-89 (220 kV) represented as UG cable (connection nodes have neglectable PF). 
The cumulated length of transmission lines remained the same in all studied cases. The proposed examples highlight the influence of the participation factor in the best way.

\section{Results and discussions}

In this section the results of the above described examples will be presented and discussed. The studied frequency ranges are up to $2 \mathrm{kHz}$ and $1.5 \mathrm{kHz}$, where capacitive coupling in transformer is ignored, for the ten-node system and for the Swiss EHV power transmission system, respectively.

\subsection{Ten-Node System}

The simulation results for the ten-node system in the four cases stated above are shown on Fig. 4.

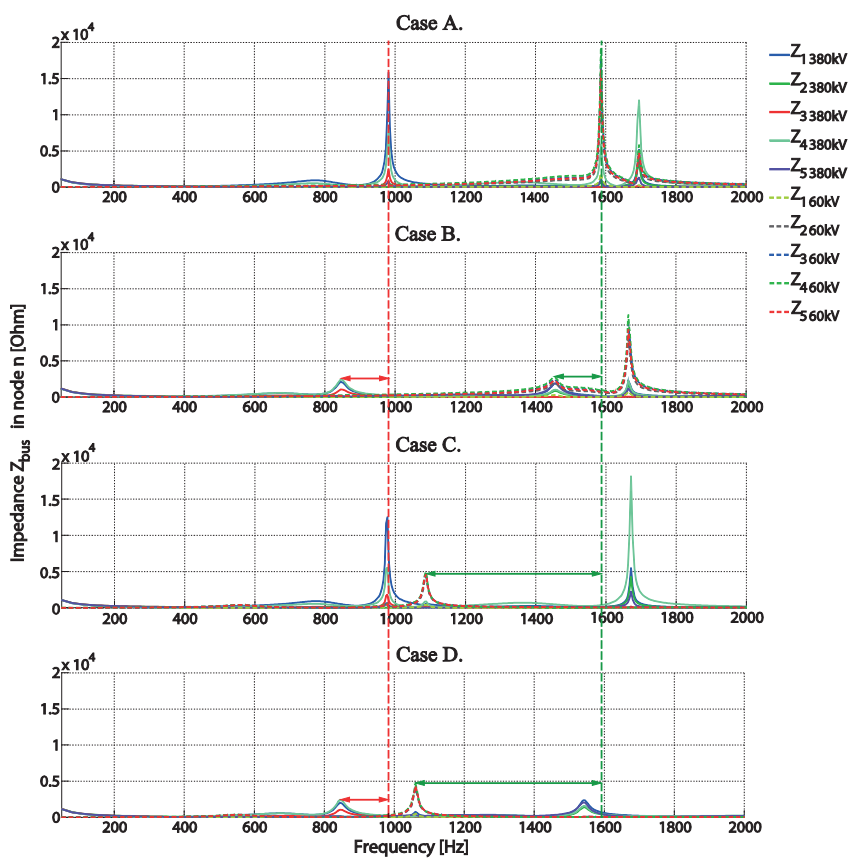

Fig. 4 Ten-node system, self-impedance as a function of frequency

As seen from the figure, the resonance peaks in cases with a power system comprising underground cables have shifted to the left compared to the case of a power system with solely overhead lines. It is also seen that the utilization of underground cables in the $60 \mathrm{kV}$ voltage level network (case C) affects mainly the frequency peaks in which participate nodes of this voltage level. However, the utilization of underground cables in the $380 \mathrm{kV}$ voltage level network (case B) affects the frequency peaks of both voltage levels.

The mutual impact of voltage levels on each other was also investigated. For this purpose a 1 A current injection was simulated in node $1(380 \mathrm{kV})$ at the nominal frequency, and at the frequencies of first two resonance peaks for each studied case. The results are shown on Fig. 5. One can see that the injection of the current at the frequency of the first resonance peak produces voltage in all nodes of the power system. The injection of $1 \mathrm{~A}$ at the frequency of the second resonance peak leads to a very high voltage generated in $60 \mathrm{kV}$ network. This can be explained by the fact that the second resonance peak is originally provoked in the $60 \mathrm{kV}$ network.

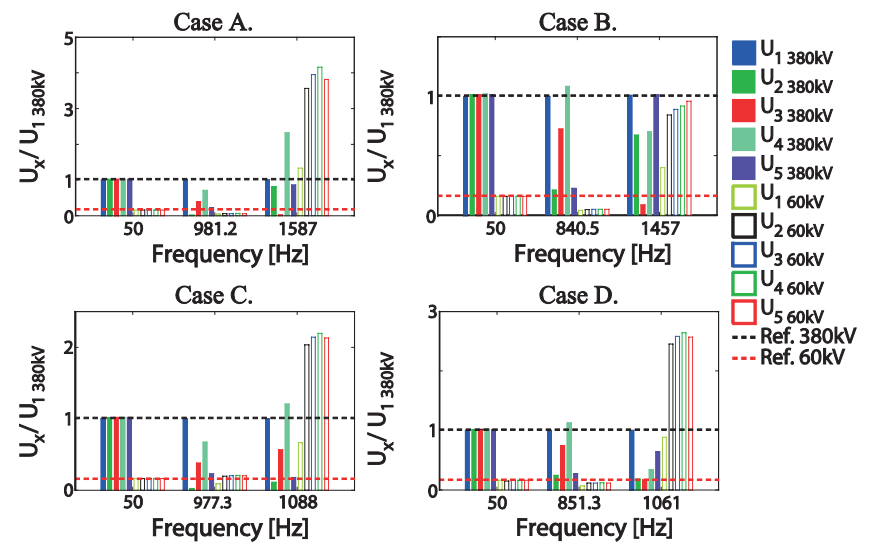

Fig. 5 Ten-node system, impact of voltage level $1(380 \mathrm{kV})$ on voltage level $2(60 \mathrm{kV})$

\subsection{Swiss EHV Power Transmission System}

The simulation of the actual Swiss EHV power transmission system (base case) was performed and the result of the frequency scan is shown on Fig. 6. Fig. 7 presents the results of RMA for each case.

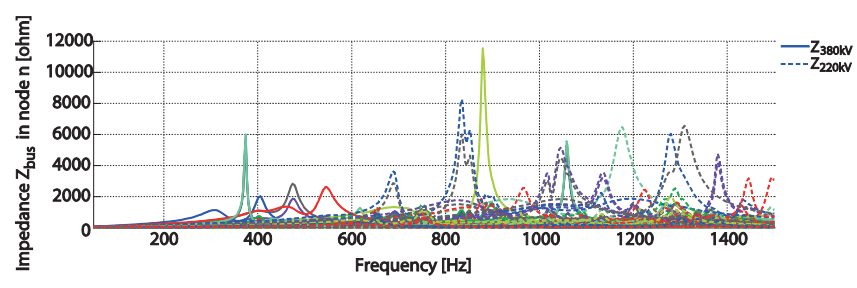

Fig. 6 Swiss EHV power transmission system, selfimpedance as a function of frequency

The participation factors (PF) were calculated for each bus at each studied frequency. In this work we focus on the resonance peak at $750 \mathrm{~Hz}$ (case E) since various nodes are participating in it.

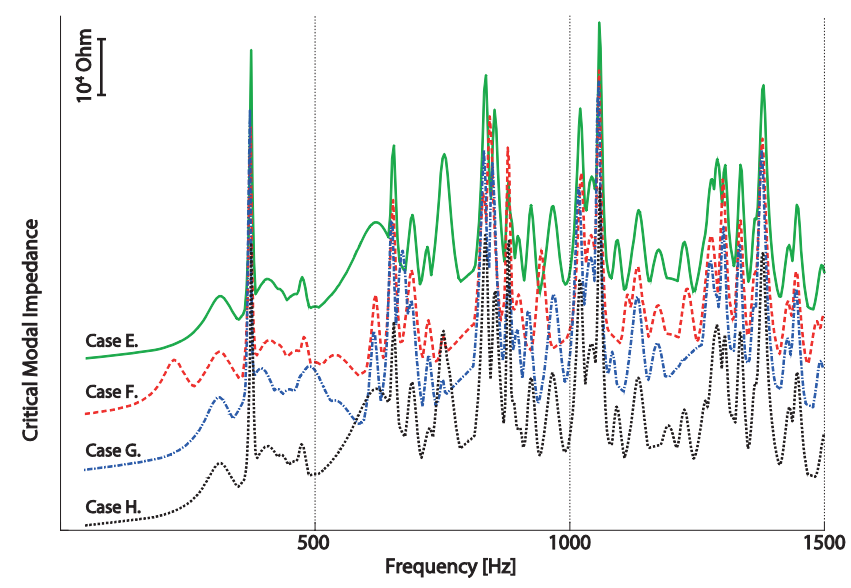

Fig. 7 Swiss EHV power transmission system, resonance mode analysis (for clarity, curves are arbitrary shifted vertically) 
Table 1 presents the values of the PF of the nodes involved in the $750 \mathrm{~Hz}$ resonance peak ( $\mathrm{PF}>0.003)$. It is therefore possible to illustrate the impact of undergrounding of transmission system at different locations of the system (nodes with high or low PF). The frequency scan results of nodes participating in the resonance peak at $750 \mathrm{~Hz}$ are presented on Fig. 8 case E.

Table 1 Participation factors at $750 \mathrm{~Hz}$

\begin{tabular}{cc|cc|cc}
\hline $\begin{array}{c}\text { Node } \\
\text { Number }\end{array}$ & PF & $\begin{array}{c}\text { Node } \\
\text { Number }\end{array}$ & PF & $\begin{array}{c}\text { Node } \\
\text { Number }\end{array}$ & PF \\
\hline $25_{380 \mathrm{kV}}$ & 0.0032 & $37_{220 \mathrm{kV}}$ & 0.0452 & $85_{220 \mathrm{kV}}$ & 0.0150 \\
\hline $28_{380 \mathrm{kV}}$ & 0.0036 & $42_{220 \mathrm{kV}}$ & 0.0495 & $91_{220 \mathrm{kV}}$ & 0.0062 \\
\hline $2_{220 \mathrm{kV}}$ & 0.0095 & $51_{220 \mathrm{kV}}$ & 0.0042 & $100_{220 \mathrm{kV}}$ & 0.0493 \\
\hline $11_{220 \mathrm{kV}}$ & 0.0052 & $55_{220 \mathrm{kV}}$ & 0.0030 & $105_{220 \mathrm{kV}}$ & 0.0225 \\
\hline $14_{220 \mathrm{kV}}$ & 0.0031 & $60_{220 \mathrm{kV}}$ & 0.0042 & $124_{220 \mathrm{kV}}$ & 0.0247 \\
\hline $17_{220 \mathrm{kV}}$ & 0.0257 & $68_{220 \mathrm{kV}}$ & 0.0044 & $125_{220 \mathrm{kV}}$ & 0.0142 \\
\hline $19_{220 \mathrm{kV}}$ & 0.0044 & $71_{220 \mathrm{kV}}$ & 0.0265 & $127_{220 \mathrm{kV}}$ & 0.0452 \\
\hline $25_{220 \mathrm{kV}}$ & 0.0107 & $78_{220 \mathrm{kV}}$ & 0.0036 & $130_{220 \mathrm{kV}}$ & 0.0227 \\
\hline $26_{220 \mathrm{kV}}$ & 0.0065 & $81_{220 \mathrm{kV}}$ & 0.0569 & $131_{220 \mathrm{kV}}$ & 0.0228 \\
\hline $31_{220 \mathrm{kV}}$ & 0.0254 & $82_{220 \mathrm{kV}}$ & 0.0045 & & \\
\hline
\end{tabular}
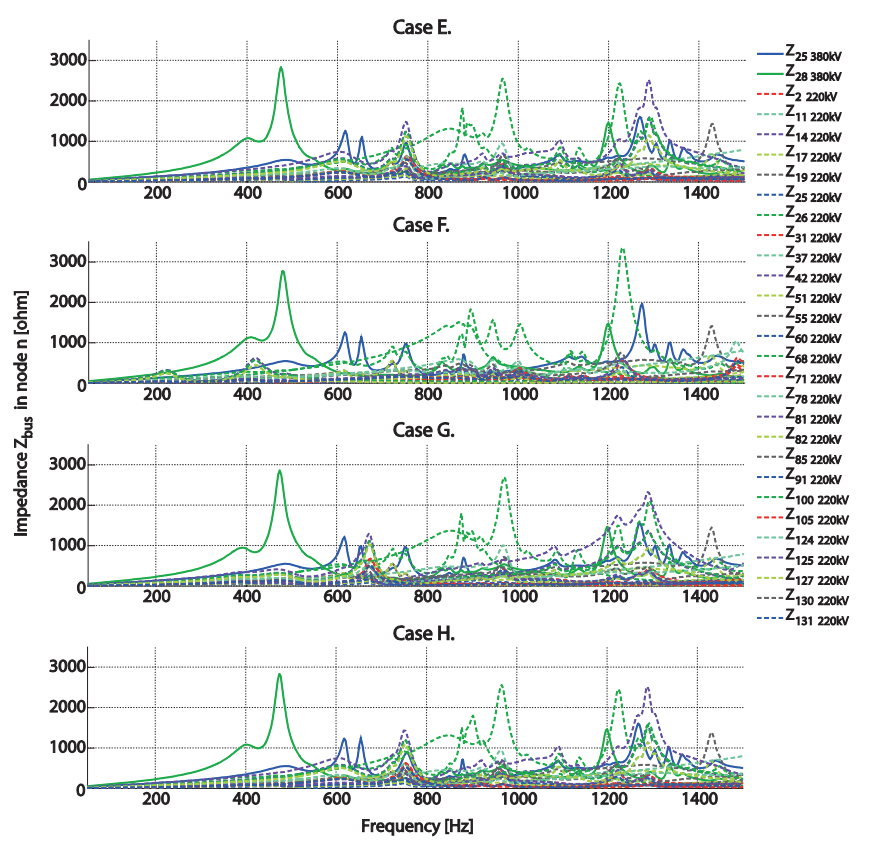

Fig. 8 Swiss EHV power transmission system, self-impedance in function of frequency

Node $81(220 \mathrm{kV})$ has the highest PF at $750 \mathrm{~Hz}$. There is only one transmission line connected to this node, it is line 81-100. The results of the frequency scan and of RMA for case F (line 81-100 is an underground cable) are shown on Fig. 8 Case F and Fig. 7. The resonance peak common for all considered nodes was at $750 \mathrm{~Hz}$ in the base case and in case $\mathrm{F}$ this resonance peak moved toward lower frequencies $(420 \mathrm{~Hz})$. Figure 8 Case $G$ presents the result for frequency scan when the line 31-71 is an underground cable (case G). As in case F, in case $G$ the resonance peak is at a lower frequency compared to the case $\mathrm{E}$ (670 $\mathrm{Hz}$ ) however the frequency change is not as dramatic as in case F. In case $H$, the line between nodes 23 and 89 is changed to an underground cable. Both of the nodes have very low participation factor $(<0.0001)$ at the frequency of $750 \mathrm{~Hz}$, actually they do not participate significantly in any of the resonances in the studied frequency interval (up to $1500 \mathrm{~Hz}$ ). The results for this case are shown in Fig. 8 case H. As could be expected from the low PF, the resonance peak remains at the same frequency as in the base case $(750 \mathrm{~Hz})$. Furthermore the results of the RMA of the base case and case $\mathrm{G}$ are almost identical.

\section{Conclusion}

In this work the frequency dependent model of a transmission system was implemented. Two methods, frequency scan and resonance mode analysis, were applied. The model was tested on two examples: the ten-node system and the Swiss power transmission system. Four simulations were performed for each example: case of the actual power system comprising solely overhead lines and three cases of mixed power systems with underground cables.

It is shown that the introduction of underground cables in a power transmission system can lead to the reduction of the resonance frequency. The participation factors of all nodes are determined. It is illustrated that undergrounding of the transmission line between nodes with high participation factors leads to a dramatic resonance frequency drop. On the other hand the transmission lines between the nodes with participation factor close to zero can be replaced by underground cables without considerable changes in the frequency response of the power transmission system.

The impact of one voltage level on another was also examined. It can be deduced that current injection in the node of one voltage level has a significant effect on the nodes of second voltage level especially at a frequency corresponding to a resonance peak provoked by one of the nodes of the second voltage level.

To conclude, the presented frequency analysis enables identification of weak nodes in a power transmission system and provides valuable information to avoid resonance frequency drop when undergrounding transmission lines in the system. To achieve better simulation results further refinements in the modeling of frequency dependent components and the inclusion of harmonic-sources like AC/DC convertors in the model are necessary. The data acquired from the real power system components could help to quantify the potential risk of resonance in a better way. 


\section{Acknowledgement}

This work is supported by EOS Holding, the Swiss Competence Centre on Energy Research „FURIES” and the University of applied sciences and arts of western Switzerland.

The authors gratefully acknowledge W. Sattinger, E. Marthe, E. Romero, SwissGrid, M. Grangier and Y. Ruckstuhl, Groupe E for their interest in our research work and their valuable contributions.

\section{References}

[1] Aeberhard, M., Vollenwyder, R., Haag, C., Aeberhardt, B. "Resonanzproblematik im SBB Energienetz." (Resonance problems in SBB transmission grid.) Technical report. [Online] Available from: http://www. news.admin.ch/NSBSubscriber/message/attachments/34330.pdf 2012 [Accessed: 29th July 2015] (in German)

[2] Meijer, S., De Jong, J. P. W., Smit, J. J., Tuinema, B. W., Lugschitz, H., Svejda, G. "Availability and risk assessment of $380 \mathrm{kV}$ cable system in transmission grids." CIGRE. 2012.

[3] METSCO Energy Solutions "Comparison of Underground and Overhead Transmission Options in Iceland (132 and 220kV)." Technical report. 2013.

[4] ENTSO-E and Europacable "Joint paper: Feasibility and technical aspects of partial undergrounding of extra high voltage power transmission lines." Technical report. Brussels. 2010.

[5] Xu, W., Huang, Z., Cui, Y., Wang, H. "Harmonic resonance mode analysis." IEEE Transactions on Power Delivery. 20 (2). pp. 1182-1190. 2005. DOI: 10.1109/tpwrd.2004.834856

[6] Cui, Y., Xu, W. "Harmonic resonance mode analysis using real symmetrical nodal matrices." IEEE Transactions on Power Delivery. 22 (3). pp. 1989-1990. 2007. DOI: 10.1109/tpwrd.2007.899481

[7] Cui, Y., Wang, X. "Modal frequency sensitivity for power system harmonic resonance analysis." IEEE Transactions on Power Delivery. 27 (2). pp. 1010-1017. 2012. DOI: 10.1109/tpwrd.2012.2185520
[8] Amornvipas, C., Hofmann, L. "Resonance analysis in transmission system: Experience in Germany." In: Power and Energy Society General Meeting, 2010 IEEE. pp.1-8, 25-29 July 2010. DOI: 10.1109/pes.2010.5588098

[9] Bollen, M. H. J., Gargari, S. M. "Harmonic resonance due to transmission cables." CIGRE. 2014.

[10] Chien, C. H., Bucknall, R. "Analysis of harmonics in subsea power transmission cables used in VSC-HVDC transmission systems operating under steady-state conditions." IEEE Transactions on Power Delivery. 22 (4). pp. 2489-2497. 2007. DOI: 10.1109/tpwrd.2007.905277

[11] Mofizul, I. S., Coates, K. M., Ledwich, G. "Identification of high frequency transformer equivalent circuit using Matlab from frequency domain data." In: Industry Applications Conference, 1997. Thirty-Second IAS Annual Meeting, IAS '97., Conference Record of the 1997 IEEE, Vol. 1, pp. 357-364, 5-9 Oct 1997. DOI: 10.1109/ias.1997.643049

[12] Morched, A., Mad, L., Ottevangers, J. "A high frequency transformer model for the EMTP." IEEE Transactions on Power Delivery. 8 (3). pp. 1615-1621. 1993. DOI: 10.1109/61.252688

[13] "Modeling and simulation of the propagation of harmonics in electric power networks. I. Concepts, models, and simulation techniques." IEEE Transactions on Power Delivery. 11 (1). pp. 452-465. 1996. DOI: $10.1109 / 61.484130$

[14] Das, J. C. "Power System Analysis: Short-Circuit Load Flow and Harmonics." 2nd Ed. New York: CRC Press, Taylor \& Francis Group. 2012.

[15] Acha, E., Madrigal, M. "Power Systems Harmonics: Computer Modelling and Analysis." New York: Wiley. 2001.

[16] Rivas, R. A. "Overhead Transmission Lines and Undeground Cables." In: Handbook of Electric Power Calculations. 3d Ed., pp. 9.1-9.33. New York: The McGraw-Hill Companies. 2001.

[17] Arrillaga, J., Watson, N. "Power system harmonics." 2nd Ed., Wiley, Christchurch. 2003. 